\title{
Mesenchymal stem cells attenuate blood- brain barrier leakage after cerebral ischemia in mice
}

\author{
Zhuo Cheng ${ }^{1}$, Liping Wang ${ }^{2}$, Meijie Qu², Huaibin Liang ${ }^{2}$, Wanlu Li', Yongfang Li ${ }^{2}$, Lidong Deng ${ }^{1}$,
} Zhijun Zhang ${ }^{1}$ and Guo-Yuan Yang ${ }^{1,2^{*}}$

\begin{abstract}
Background: Ischemic stroke induced matrixmetallo-proteinase-9 (MMP-9) upregulation, which increased bloodbrain barrier permeability. Studies demonstrated that mesenchymal stem cell therapy protected blood-brain barrier disruption from several cerebrovascular diseases. However, the underlying mechanism was largely unknown. We therefore hypothesized that mesenchymal stem cells reduced blood-brain barrier destruction by inhibiting matrixmetallo-proteinase-9 and it was related to intercellular adhesion molecule-1 (ICAM-1).

Methods: Adult ICR male mice $(n=118)$ underwent 90-min middle cerebral artery occlusion and received $2 \times 10^{5}$ mesenchymal stem cell transplantation. Neurobehavioral outcome, infarct volume, and blood-brain barrier permeability were measured after ischemia. The relationship between myeloperoxidase (MPO) activity and ICAM-1 release was further determined.

Results: We found that intracranial injection of mesenchymal stem cells reduced infarct volume and improved behavioral function in experimental stroke models $(p<0.05)$. lgG leakage, tight junction protein loss, and inflammatory cytokines IL-1 $\beta, I L-6$, and TNF-a reduced in mesenchymal stem cell-treated mice compared to the control group following ischemia $(p<0.05)$. After transplantation, MMP-9 was decreased in protein and activity levels as compared with controls $(p<0.05)$. Furthermore, myeloperoxidase-positive cells and myeloperoxidase activity were decreased in mesenchymal stem cell-treated mice $(p<0.05)$.

Conclusion: The results showed that mesenchymal stem cell therapy attenuated blood-brain barrier disruption in mice after ischemia. Mesenchymal stem cells attenuated the upward trend of MMP-9 and potentially via downregulating ICAM-1 in endothelial cells. Adenosine 5'-monophosphate (AMP)-activated protein kinase (AMPK) pathway may influence MMP-9 expression of neutrophils and resident cells, and ICAM-1 acted as a key factor in the paracrine actions of mesenchymal stem cell.
\end{abstract}

Keywords: Blood-brain barrier, ICAM-1, Inflammation, Matrixmetallo-proteinase-9, Ischemia, Mesenchymal stem cell

\section{Background}

Ischemic stroke has been identified as the second leading cause of disability and death behind ischemic heart disease worldwide [1]. In China, there are 2.4 million new strokes and 1.1 million stroke-related deaths each

\footnotetext{
* Correspondence: gyyang@sjtu.edu.cn

${ }^{1}$ School of Biomedical Engineering and Shanghai Jiao Tong University affiliated sixth people's hospital, Shanghai Jiao Tong University, Shanghai 200000, China

${ }^{2}$ Department of Neurology, Ruijin Hospital, School of Medicine, Shanghai Jiao Tong University, Shanghai 200025, China
}

year [2]. Reducing high mortality and morbidity could therefore benefit stroke patients, family caregivers, and society in burden alleviation.

Matrixmetallo-proteinases are a family of zinc and calcium-dependent endopeptidases, which is capable to degrade all components of extracellular matrix (ECM) including laminin, collagen, and fibronectin surrounding the blood-brain barrier (BBB) [3]. BBB leakage is a key issue in the injury cascade, which exacerbates ischemic brain injury. Through peripheral immune cells into the brain to enhance the neuroinflammatory response,

(c) The Author(s). 2018 Open Access This article is distributed under the terms of the Creative Commons Attribution 4.0 International License (http://creativecommons.org/licenses/by/4.0/), which permits unrestricted use, distribution, and 
vasogenic edema comes to hasten $[4,5]$. Clinical and experimental studies demonstrated that matrixmetalloproteinase (MMP-2, MMP-3, MMP-7, or MMP-9) was activated and upregulated after ischemic stroke [6-10]. MMP-2-induced early BBB disruption is deemed reversible since the tight junction components loosen and they remain in the endothelial cleft. Thus, BBB can be reassembled. In contrast, MMP-9 is markedly elevated in the late phase of BBB disruption, which occurs at 24-72 $\mathrm{h}$ after ischemic stroke [11]. MMP-9 is a 92-kDa type IV collagenase, which is also called gelatinase B. MMP-9 breaks tight junction proteins (e.g., occludin and claudin-5) and basal lamina proteins (e.g., fibronectin, laminin, collagen), resulting in barrier disruption in physiological process. It is noted that inhibition of MMP-9 provided robust protection against the $\mathrm{BBB}$ disruption [12], suggesting that MMP-9 was the dominant protease acting at the $\mathrm{BBB}$ following ischemic stroke [13].

In brain, MMPs are expressed by various cell types including resident cells such as endothelial cells, microglia, neurons, astrocytes, and infiltrated inflammatory cells during ischemic stroke $[14,15]$. Neutrophils are the main inflammatory cell type that responded to the inflammatory stimulus following ischemic stroke [16]. It is interested that myeloperoxidase, a major component of neutrophil azurophilic granules, is MMP-9 postive, suggesting that neutrophils are the main source of MMP-9 following ischemic stroke [17]. Transmigration of neutrophils was thought to be reliant.

Mesenchymal stem cells (MSCs), a subset of nonhematopoietic stem cells residing in bone marrow, support the growth and differentiation of hematopoietic stem cells and possibly repopulate stem cells in other tissues [18]. Systemic administration of MSCs after cerebral infarction could reduce infarct volume and improve behavioral function in experimental stroke models [19, 20]. MSCs exhibit potent immunosuppressive activity [21]. Meanwhile, they have a potential to maintain BBB integrity [22]. Our previous study showed that MSCs could protect $\mathrm{BBB}$ integrity by reducing the astrocyte apoptosis after ischemic attack, which was due to the attenuation of inflammatory response and downregulation of aquaporin-4 expression [22]. However, limited studies focused on the effect of MSCs on MMP-9 activity via transmigration of neutrophils.

In this research, we explored (1) whether MSC therapy attenuates $\mathrm{BBB}$ breakdown and reduces inflammatory response following transient MCAO in mice, (2) whether the effect of protection related to MMP-9 and how MSCs contribute to the attenuation of MMP-9 increase during transient MCAO in mice, and (3) what molecular signaling pathways involve in MSC therapy.

\section{Methods}

\section{Experimental design}

Animal protocol was approved by the Institutional Animal Care and Use Committee of Shanghai Jiao Tong University, Shanghai, China. All animal procedures were performed to minimize pain or discomfort in accordance with current protocols. Adult male ICR mice $(n=118)$ weighing 30-32 g were divided into four groups: (1) MSC treated $(n=36)$, (2) PBS treated $(n=55)$, (3) MMP9 inhibitor (SB-3CT, $n=12)$, and (4) sham $(n=15)$ group. At 1 and 3 days following transient middle cerebral artery occlusion (tMCAO), neurological severity score was performed before mice were sacrificed. Then, samples were collected for further study.

The oxygen-glucose deprivation (OGD) experiment was performed using four groups differentiated as follows: (1) bEND.3 cells (mouse brain microvascular endothelial cells, endothelialpolyoma middle $\mathrm{T}$ antigen transformed from cerebral cortex, American Type Culture Collection (ATCC)) alone, (2) bEND.3 cells co-cultured with MSCs, (3) bEND.3 cells cultured with condition medium (CM) of MSCs, and (4) bEND.3 co-cultured with MSCs plus compound C (Sigma, St. Louis, MO). bEND.3 cells alone without oxygen-glucose deprivation was the control (ctrl).

\section{MSC isolation and identification}

Bone-derived MSCs (MSCs) were isolated and harvested from adult male Sprague Dawley rats (SD, Jiesijie, Co., Shanghai, China) weighing 200-250 g as previously described [23]. The cells were suspended in Dulbecco's modified Eagle's medium (DMEM; Gibco Laboratories, Grand Island, NY) with 10\% fetal bovine serum (FBS; Life Technologies, Carlsbad, CA) and incubated at $37^{\circ} \mathrm{C}$ with $5 \% \mathrm{CO}_{2}$. Identification was performed by flow cytometry (BD Biosciences, Mississauga, ON) following the instructions. Cells were incubated in $100 \mu \mathrm{l}$ PBS with CD29-APC (1:100 dilution, BD Biosciences, Mississauga, ON), CD90-cy5.5 (1:100 dilution, BD Biosciences), CD31-PE (1:100 dilution, eBioscience, San Diego, CA), FITC-CD45 (1:100 dilution, eBioscience), and their isotype control antibodies for $20 \mathrm{~min}$ on ice. After three washes with PBS, the cells were suspended in $300 \mu \mathrm{l}$ of PBS and analyzed in fluorescence-activated cell sorter (FACS) instrument (BD Biosciences).

\section{Transient middle cerebral artery occlusion (MCAO)}

After anesthetizing mice with ketamine/xylazine (100 mg/10 mg/kg, Sigma, St. Louis, MO) intraperitoneally, the occlusion of middle cerebral artery (MCA) was achieved by inserting a silicone-coated 6-0 nylon suture (Covidien, Saint Louis, MA) from the incision on left external carotid artery (ECA) with an advancement of 9-10 mm toward MCA. Successful occlusion of MCA was confirmed using a laser Doppler flowmetry (Moor 
Instruments, Devon, UK) as a decline in the regional blood flow by more than $80 \%$ compared to the contralateral hemisphere. The suture was withdrawn completely, and reperfusion was achieved after 90 min of transient MCAO. The mortality in our study was less than $10 \%$.

\section{Oxygen-glucose deprivation}

bEND.3 cells were seeded in six-well plates and incubated with FBS-free condition medium (Gibco) at 5\% $\mathrm{CO}_{2}$ and $95 \% \mathrm{~N}_{2}$ atmosphere using an airtight chamber for $6 \mathrm{~h}$ followed by re-oxygenation as previously described [24].

\section{Neurobehavioral assessments}

Neurobehavioral assessments were conducted by an experiment partner who was blind to the treatment conditions. For neurological function assessment, a modified Neurological Severity Scores (mNSS) ranging from 0 to 14 score was adopted, which included raising the mouse by the tail ( 0 to 3$)$, walking on the floor (0 to 3$)$, beam balance tests (0 to 6 ), and the response absence (0 to 2 ).

\section{MSC transplantation and labeling}

For cellular tracking after transplantation, cells were labeled with carboxyfluorescein diacetate-succinimidyl ester (CFDA-SE, Beyotime, Shanghai, China). MSCs were resuspended in $1 \mathrm{mM}$ CFDA dye at $37^{\circ} \mathrm{C}$ for $20 \mathrm{~min}$. The animals received stereotaxic transplantation within $15 \mathrm{~min}$ after reperfusion via a 10-ml Hamilton syringe (Hamilton, Bonaduz, Switzerland) injection. The transplant coordinate was $2 \mathrm{~mm}$ lateral to the sagittal suture and $1 \mathrm{~mm}$ posterior to the coronal suture and $3 \mathrm{~mm}$ under the dura which is in peri-ischemic area. We injected MSC suspension with $1 \times 10^{5}$ cells $/ 5 \mu \mathrm{l}$ potassium phosphate (PBS) buffer at a rate of $0.5 \mu \mathrm{l} / \mathrm{min}$.

As a positive control, 2-[((4-phenoxyphenyl) sulfonyl]methyl]-thiirane (SB-3CT; Sigma), an inhibitor of MMP9, was also used. It was diluted in $10 \%$ dimethylsulfoxide/ $90 \% \mathrm{NS}$ and was injected intraperitoneally at $10 \mathrm{mg} / \mathrm{kg}$ per day for 3 consecutive days beginning from days 1 to 3 after ischemia.

\section{Infarct volume measurement}

Infarct volume was measured using Cresyl Violet acetate (Sigma) staining. Sections $(200 \mu \mathrm{m}$ apart) were obtained from all brain tissue including infarct area. The ischemic area of each section was depicted by image and measured by Image $J(\mathrm{NIH}$, Bethesda, MD, https://imagej. nih.gov/ij/). The contralateral area minus the normal area of the ipsilateral hemisphere was recorded as the infarct area $\Delta \mathrm{S}$. The infarction area of two adjacent pieces are denoted as $\Delta S 1$ and $\Delta S 2$; the volume of infarction $(V)$ for two adjacent cerebral volume is one third of thickness $(H) \times(\Delta \mathrm{S} 1+\Delta \mathrm{S} 2+\sqrt{ }(\mathrm{S} 1 \times \mathrm{S} 2))$; the thickness $(H)=0.2 \mathrm{~mm}$. Then, sum all cerebral infarction volume of two adjacent brain tissues.

\section{Immunohistochemistry}

Frozen sections were fixed with methanol for $10 \mathrm{~min}$ and incubated in $0.3 \%$ Triton X-100 solution for $10 \mathrm{~min}$ and blocked with $10 \%$ bovine serum albumin (BSA) for $1 \mathrm{~h}$. After blocking, the sections were incubated with primary antibodies against ZO-1 (1:200, Invitrogen, Carlsbad, CA), occuldin (1:200, Invitrogen), claudin-5 (1: 200, Invitrogen), and CD31 (1:200; R\&D Systems, Minneapolis, MN) overnight at $4{ }^{\circ} \mathrm{C}$ under humidified condition. After washing with PBS, sections were incubated with secondary antibody for $2 \mathrm{~h}$ at $37{ }^{\circ} \mathrm{C}$ to perform immunofluorescence. After closure, get observation under confocal microscope (Leica, Solms, Germany).

$\mathrm{DAB}$ staining for IgG and myeloperoxidase (MPO): brain sections were incubated in $0.3 \% \mathrm{H}_{2} \mathrm{O}_{2}$ in methanol for $30 \mathrm{~min}$. The primary antibody MPO (1:300 dilution, R\&D Systems) were incubated overnight at $4{ }^{\circ} \mathrm{C}$ and incubated with Universal ABC Kit (Vector Laboratories). The reaction product was visualized using a $\mathrm{DAB}$ peroxidase substrate (Vector Laboratories, Burlingame, CA). Sections were analyzed using bright-field microscopy (Leica). Mean optical density was measured using Image-Pro Plus software (Media Cybernetics, Bethesda, MD, www.mediacy.com). The value of integrated optical density (IOD) after optical density correction and the area of interested was used for the quantitative analysis of IgG.

\section{Western blot analysis}

Tissue from peri-ischemic areas was placed in RIPA Lysate (Millipore, Bedford, MA) with protease inhibitor cocktail (Thermo), phosphatase inhibitors (Thermo), and phenylmethyl sulfonyl fluoride (PMSF, Thermo) in ice for $30 \mathrm{~min}$ and was centrifuged at $12000 \mathrm{rpm}$ for 15 min at $4{ }^{\circ} \mathrm{C}$. The supernatant was obtained from the centrifuged mixture and stored at $-80{ }^{\circ} \mathrm{C}$. The protein concentration of stored samples was measured using the BCA Protein Assay Kit (Thermo).

The samples were subjected to SDS-polyacrylamide gel electrophoresis and transferred to filter membrane. The membranes were blocked with $5 \%$ non-fat milk and incubated with primary antibody MMP-9 (1:1000 dilution, Millipore), ICAM-1 (1:1000 dilution, R\&D Systems), and AMPK/p-AMPK (1:1000 dilution, Cell Signaling Technology, Beverly, MA) overnight. $\beta$-Actin (1:1000 dilution, Cell Signaling Technology) was employed as the loading control. The blots were incubated with the appropriate horseradish peroxidase (HRP)-conjugated secondary antibody after washing with Tris-buffered saline. Immunoblots were detected using an enhanced chemiluminescence (ECL) kit (FD 
Technology, Shanghai, China) and calculated using Image $J$ software (NIH, Bethesda, MD).

\section{Real-time PCR}

Total RNA extraction and real-time PCR were performed according to the manufacturer's instructions. Total RNA was extracted from tissues around the lesional sites 1 and 3 days after transient MCAO using Trizol reagent (Invitrogen). Total RNA was reverse transcribed to cDNA with Zymoscript first-strand cDNA synthesis kit (zymo tool, Shanghai, China). Gene transcription was detected by real-time PCR in a 7900HT sequence detection system (Applied Biosystems, Foster City, CA) using specific primers designed from known sequences. GAPDH (Cell Signaling Technology) was used as an endogenous control. Sequence-specific primers for IL- $1 \beta$, IL-6, TNF- $\alpha$, and GADPH were showed as follows:

\begin{tabular}{|c|c|c|c|}
\hline ne & Forward primer $\left(5^{\prime}-3^{\prime}\right)$ & Reverse primer $\left(5^{\prime}-3^{\prime}\right)$ & $\mathrm{bp}$ \\
\hline & SGA & TT & 88 \\
\hline & $C T$ & ITGGGGTCCGTCAACT & \\
\hline a & ATG & $\mathrm{GGC}$ & \\
\hline DPH & AGGTCGGTGTGAACGGATTTG & TGTAGACCATGTAGTTGAGGTCA & \\
\hline
\end{tabular}

\section{MPO activity assay}

MPO activity assay was performed as described previously [24]. In brief, $10 \mu \mathrm{l}$ brain protein of which had diluted as 1:10 from ipsilateral hemisphere was added to $180 \mu \mathrm{l}$ of work solution containing $2 \mathrm{mmol} / \mathrm{l}$ O-dianisidin-dihydrochloride (Sigma) dissolved in
$50 \mathrm{mmol} / \mathrm{l} \mathrm{PBS}(\mathrm{pH}=6)$. Before measurement, $10 \mu \mathrm{l}$ of $100 \mathrm{mmol} / \mathrm{l} \mathrm{H}_{2} \mathrm{O}_{2}$ was added. Changes in absorbance at $460 \mathrm{~nm}$ over $10 \mathrm{~min}$ were measured.

\section{Zymography}

Dilute protein extracts with Zymogram sample buffer (BioRad, Hercules, CA). Use 10\% SDS-polyacrylamide gels containing 1\% gelatin (Sangon Biotech, Shanghai, China) to run $150 \mathrm{~V}$ for about $1 \mathrm{~h}$; float the gels in $2.5 \%$ Triton X-100 to remove the SDS. Incubate the gels for $72 \mathrm{~h}$ at $37{ }^{\circ} \mathrm{C}$ in $1 \times$ LSCB solution (10×: $0.5 \mathrm{M}$ Tris Base, $\mathrm{pH} 7.6,2.0 \mathrm{M}$ $\mathrm{NaCl}, 0.05 \mathrm{M} \mathrm{CaCl}_{2}, 0.2 \%$ (w/v) Brij-35). Stain the gels for $1 \mathrm{~h}$ in coomassie stain (Coomassie Brilliant Blue, 30\% methanol, $10 \%$ acetic acid). Store the gels in $10 \%$ acetic acid for destain gels for photography and densitometric analysis which measured the gelatinolytic activities.

\section{Statistical analysis}

Results were presented as mean \pm SD. Statistical analysis was evaluated by GraphPad Prism 5 software (San Diego, CA, https://www.graphpad.com/). For comparison between the two groups, statistical significance was determined through Student's $t$ test. For comparison among multiple groups, statistical significance was evaluated using one-way ANOVA followed by a Student-Newman-Keuls test. $p<0.05$ was considered statistically significant.

\section{Results}

MSC isolation, identification, and injection

MSCs were isolated from the femoral and tibial bone marrow of adult male SD rats. Cultured cells showed typically spindle-shaped morphology under phasecontrast microscopy (Fig. 1a). Cytometry analysis depicted that $99.99^{+} \%$ cultured cells were positive for CD29 and
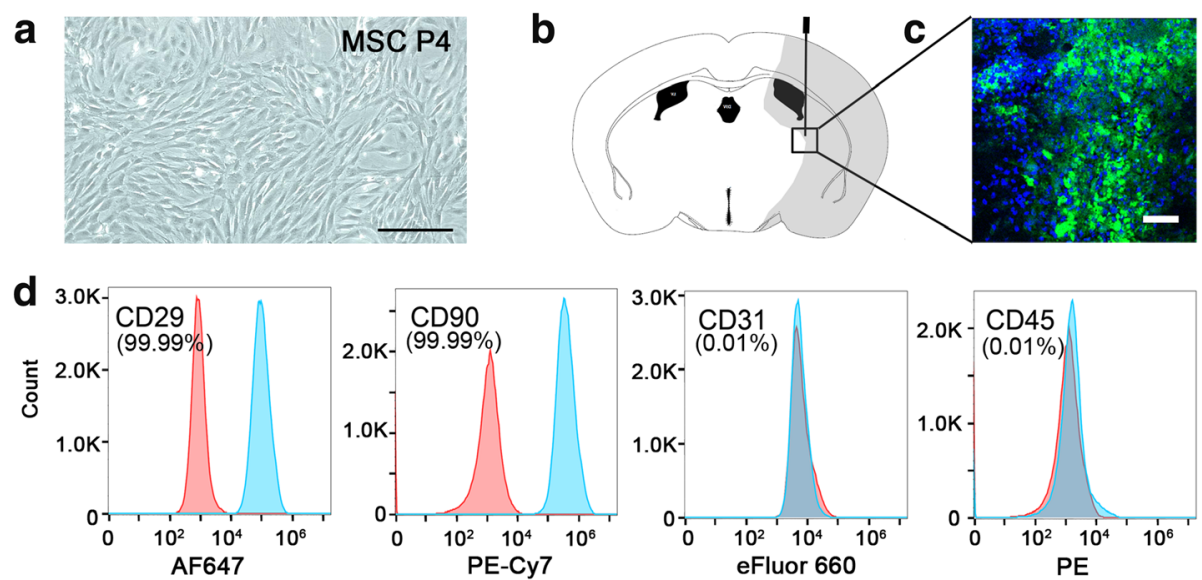

Fig. 1 Bone-derived mesenchymal stem cell (MSC) isolation, identification, and injection. a Morphology of MSCs in cell culture. Cultured cells showed typically spindle-shaped morphology under phase-contrast microscopy. $\mathbf{b}$ Schematic diagram of cell injection into the striatum of the brain and the survival of MSCs after injection. c Green fluorescent (CFDA SE dye) cells were located in the ischemic hemisphere after 3 days of injection. Scale bar $=300 \mu \mathrm{m}$. $\mathbf{d}$ Cytometry analysis depicted that $99.99^{+} \%$ cultured cells were positive for CD29 and CD90 and negative for CD31 and CD45 
CD90. By contrast, CD31 and CD45 were expressed in only $0.1 \%$ of total cells (Fig. 1d). Green fluorescent (CFDA dye)-positive cells, suggesting a number of survived transplanted cells, were located in the ischemic hemisphere after 3 days of injection (Fig. 1b, c).

\section{MSC transplantation improved neurological outcomes} and reduced stroke volume following transient MCAO

We tested the neurological outcomes at 1 and 3 days after transient MCAO using mNSS (Fig. 2c). The results showed that the neurological deficits were reduced after MSC transplantation following 1 and 3 days of transient
MCAO $(p<0.05)$. MSCs ameliorated infarct volume in the ischemic hemisphere at days 1 and 3 compared to the PBS group $(p<0.05)$ (Fig. 2a).

\section{MSC transplantation attenuated BBB breakdown following transient MCAO}

For evaluating BBB permeability, IgG staining displayed that IgG protein leaked into brain tissue after transient MCAO at days 1 and 3. Quantitative analysis revealed less IgG protein leaked into brain tissue in MSC-treated mice compared to PBS-treated mice $(p<0.05)$. MSC transplantation reduced IgG leakage at day 3 (Fig. 2b). To evaluate a
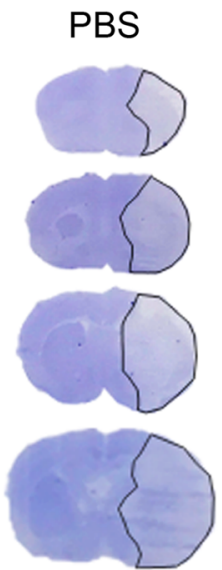

b

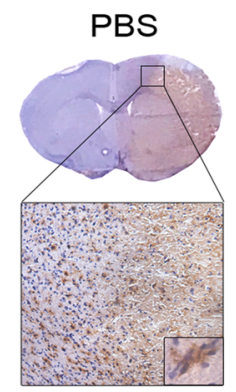

C

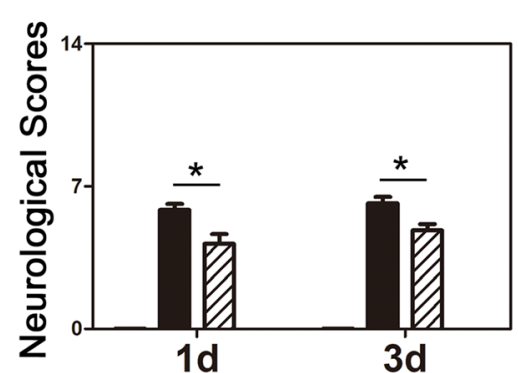

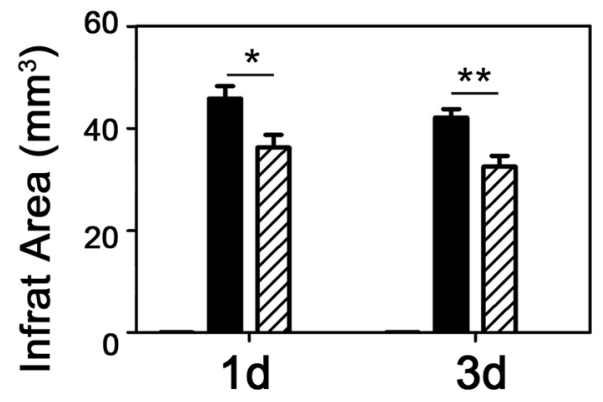

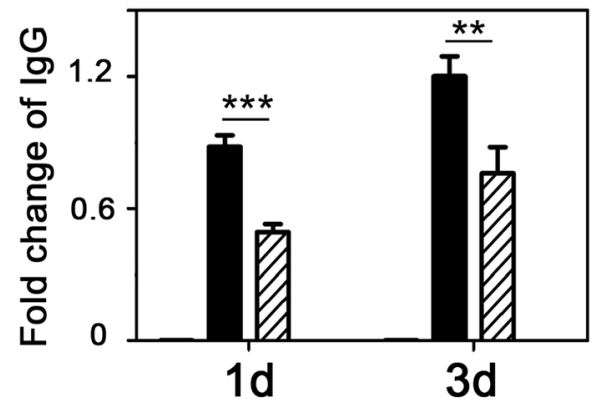

Fig. 2 MSC transplantation improved neurological outcomes and reduced infarct volume in mice via maintaining BBB integrity following transient MCAO. a Photographs showed a series of coronal sections with Cresyl Violet staining following transient MCAO in the PBS- and MSC-transplanted mice. Bar graph showed the quantification of both groups. $\mathbf{b}$ IgG staining displayed that lgG protein leaked into brain tissue in PBS- and MSC-treated mice at day 3. Quantitative analysis of leaked lgG protein revealed that less lgG protein leaked into brain tissue in MSC-treated mice compared to PBStreated mice. The images in square frames of graphs (below) were amplified as images (above), respectively. c MSCs improved neurological outcomes. MSCs significantly ameliorated neurological outcomes in the ischemic hemisphere at both days 1 and 3 compared with the PBS group. Data are mean \pm SD, $n=5-6$ per group. ${ }^{*} p<0.05,{ }^{* *} p<0.01,{ }^{* * *} p<0.001$ 
tight junction distribution in endothelial cells after MSC transplantation, we conducted immunostaining with specific cell markers: occludin/CD31, ZO-1/CD31, and claudin-5/CD31co-staining in sections from ischemic penumbra (Fig. 3). MSCs reversed gap formation of ZO-1, occludin, and claudin-5, which indicated that BBB integrity was protected by MSC treatment $(p<0.05)$.

MSC therapy reduced expression of IL- $1 \beta, \mathrm{IL}-6$, and TNF- $\alpha$ and alleviated neutrophil infiltration following MCAO

To determine whether the effect of MSCs on BBB breakdown after MCAO was involved in the immunomodulatory influence of MSCs, we examined IL-1 $\beta$, IL-6, and TNF- $\alpha$ mRNA in mice brain (Fig. 4a). We demonstrated that IL- $1 \beta$, IL- 6 , and TNF- $\alpha$ mRNA were increased at days 1 and 3 following MCAO $(p<0$. 05). Three cytokines decreased in MSC-treated group at day 3 compared to the PBS group. To investigate the neutrophil infiltration in the acute phase of cerebral ischemia, we performed DAB staining to detect MPOpositive cells (Fig. 4b). There was a decrease in MPOpositive cells at 1 and 3 days after transient MCAO in the MSC-transplanted group compared to the PBS group $(p<0.05)$. MPO activity is an indicator of neutrophil. Reduced neutrophil infiltration means reduced activity and declined function. To determine the status changes of neutrophil, MPO activity was expressed as units per milligram tissue (Fig. 4c). One unit of MPO activity represents the amount of enzyme degrading $1 \mu \mathrm{mol} \mathrm{H}_{2} \mathrm{O}_{2}$ per minute at $25^{\circ} \mathrm{C}$. It was noted that neutrophil infiltration was greatly reduced in the MSCtransplanted mice after transient MCAO $(p<0.05)$.

\section{MSCs suppressed MMP-9 upregulation after transient MCAO}

Western blot and zymography analysis of MMP-9 were performed in the PBS, MSC-treated, and SB-3CT groups (Fig. 5). We demonstrated that ischemia-reperfusion increased MMP-9 expression and this increase was attenuated after MSC transplantation, which was similar to SB-3CT, the inhibitor of MMP-9. Brain injury-induced MMP-9 upregulation contributed to BBB leakage, but MSC transplanted lowered the MMP-9 transplanted and function which inhibited the critical BBB breakdown after ischemia $(p<0.05)$.

\section{MSCs downregulated ICAM-1 expression in vivo and in vitro}

To explore the relationship between MMP-9 and ICAM-1 in vivo, we executed western blot for ICAM-1 which was related to neutrophil transmigration. Results showed the significant reduction of ICAM-1 expression in MSC-transplanted group $(p<0.05)$ (Fig. 6a). Similar result was obtained from in vitro: after $\mathrm{OGD} /$ re-oxygenation treatment, co-culture bEND.3 cells with MSCs

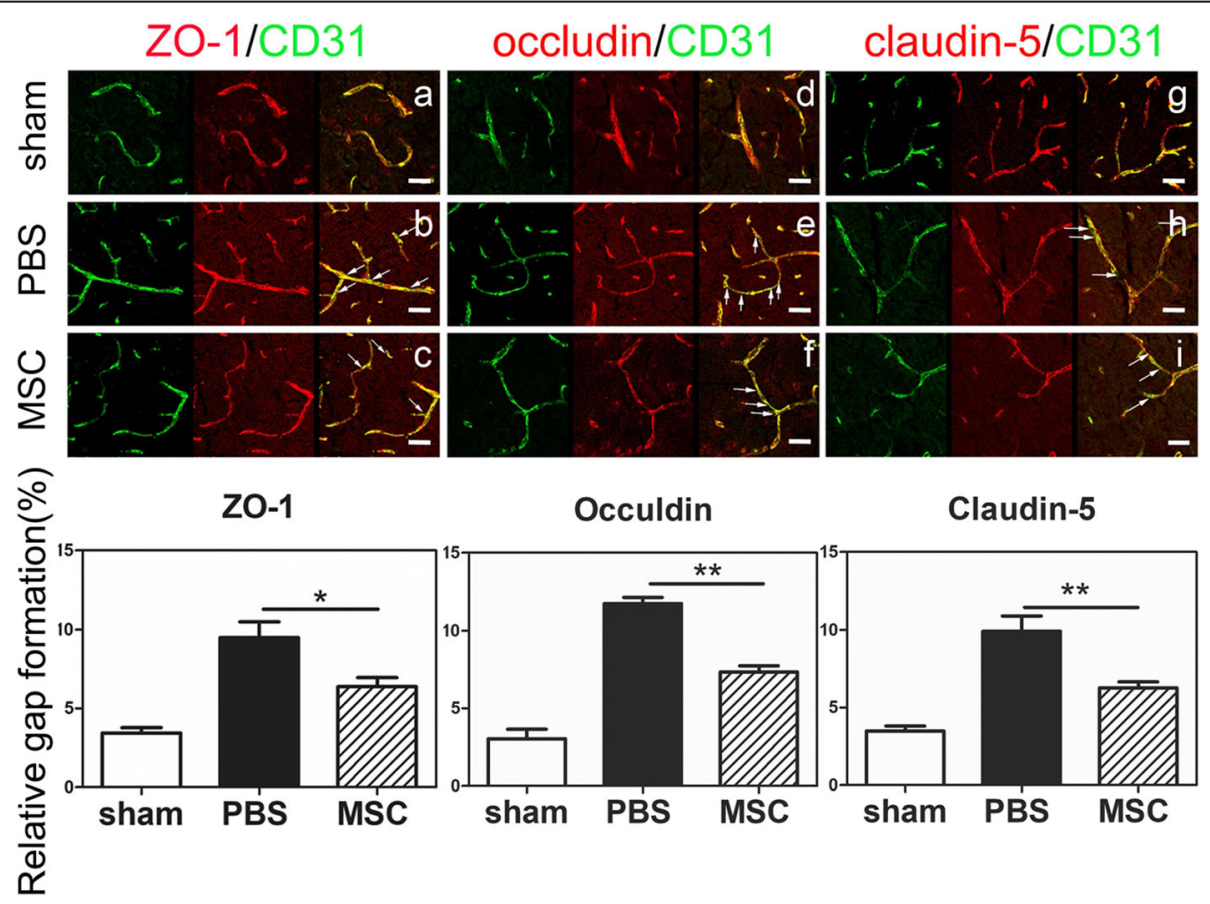

Fig. 3 MSCs reversed gap formation of ZO-1, occludin, and claudin-5. Sections from ischemic penumbra were stained of ZO-1, occludin, and claudin-5 (red) and then co-stained with endothelial marker CD31 (green). Discontinuous labeling and gap formation (white arrows) were observed in ipsilateral brains following 3 days of tMCAO. Scale bar $=30 \mu \mathrm{m}$. Data are mean $\pm S D, n=5-6$ per group. ${ }^{*} p<0.05,{ }^{* *} p<0.01,{ }^{* * *} p<0.001$ 
a
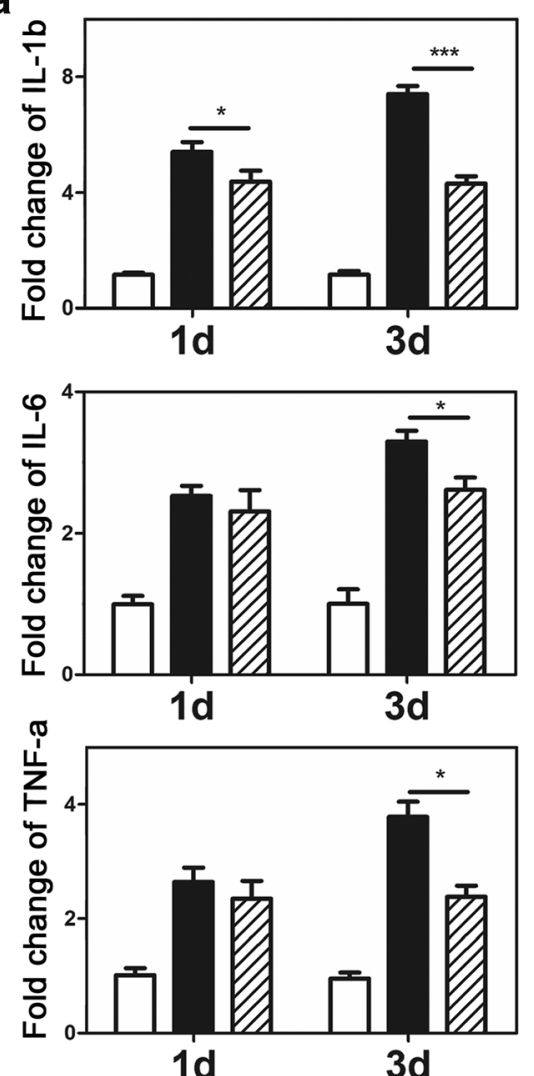

C

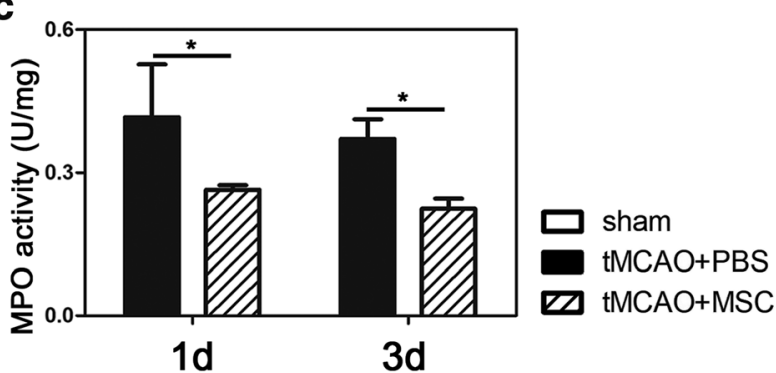

b
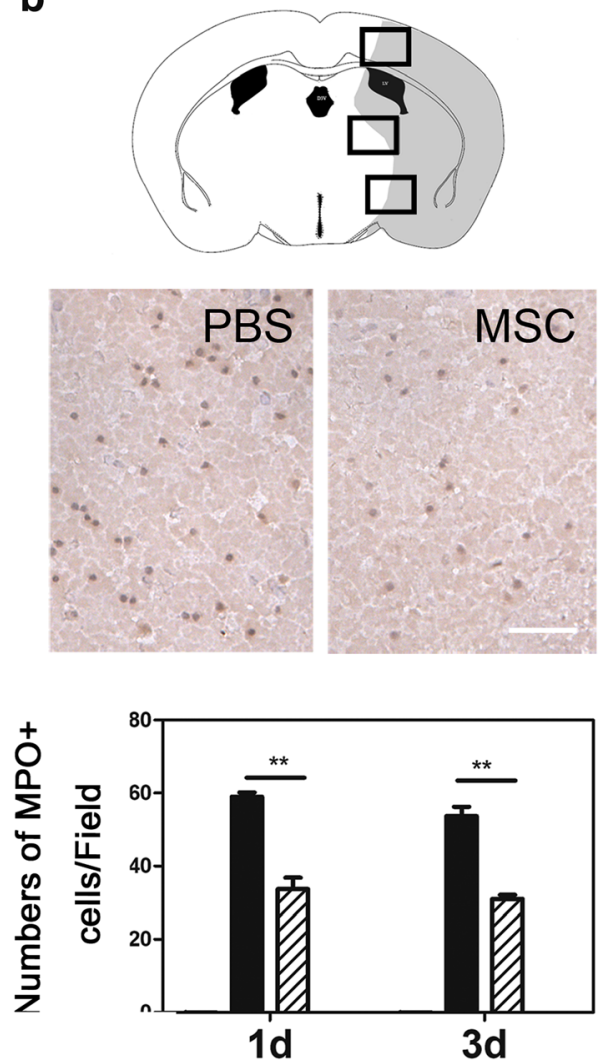

Fig. 4 MSCS alleviated neutrophil infiltration and inflammatory cytokine expression following transient MCAO. a The relative mRNA expression of IL-1 $\beta$, IL-6, and TNF-a normalized to GAPDH was measured at days 1 and 3 following MCAO. They were significantly decreased in the MSC-treated group compared to the PBS group at day 3; however, only IL-1 $\beta$ was significant at day 1. b MPO+ cells and their quantification in the PBS- and MSC-transplanted mice at 1 and 3 days following MCAO. In ischemic hemisphere, there were less MPO+ cells per field in MSC-transplanted group than PBS group, which represented less neutrophil adhesion and infiltration. Scale bar $=100 \mu \mathrm{m}$. c Bar graph showed the MPO activity in sham and PBS- and MSC-treated mice. Data are mean \pm SD, $n=5-6$ per group. ${ }^{*} p<0.05,{ }^{* *} p<0.01,{ }^{* * *} p<0.001$

displayed effective inhibition $(p<0.05)$. We further used the MSC condition medium (CM) to confirm whether it was MSC's paracrine contributed to the ICAM-1 downregulation in cultured bEND.3 cells (Fig. 6b). The experimental results were consistent with our conjecture. ICAM-1 was expressed constituently at a low level in endothelial cells, and after OGD the expression was elevated in protein level. Both MSC condition medium and co-culture could inhibit the upregulation of ICAM1 in bEND. 3 cells. ICAM- 1 acted as a key factor in the paracrine actions of MSCs.
MSCs downregulated ICAM-1 expression which could be via AMPK signaling

We performed western blot to assess the relationship between MSC-induced downregulation of ICAM-1 and the phosphorylation status of AMPK at threonine residue (Fig. 7). We analyzed the effect of MSC at a cellular level using OGD models to mimic in vivo ischemia/reperfusion injury. Results indicated that ICAM-1 was reduced in MSC-treated group compared to the PBStreated group $(p<0.05)$. To test whether AMPK pathway was involved in MSC group after OGD, we also used a 


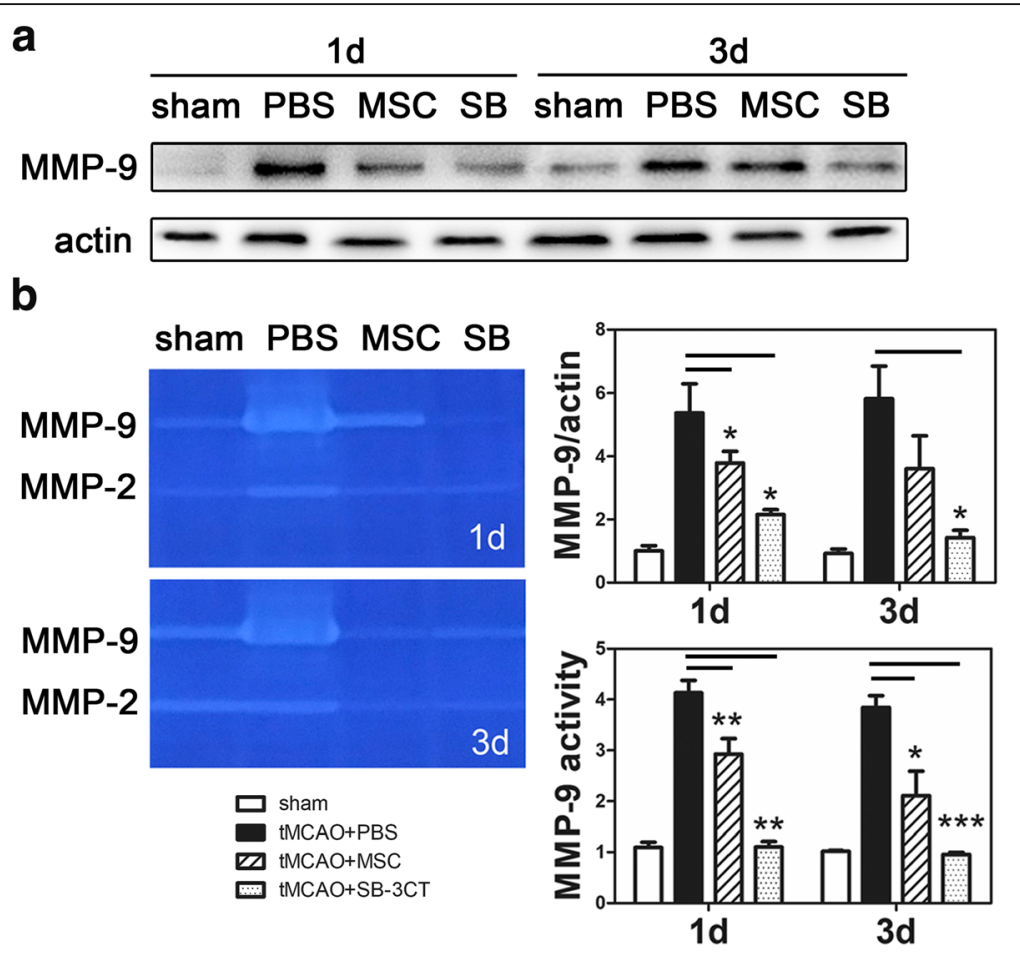

Fig. 5 MSCs significantly inhibited MMP-9 upregulation in protein level and activity at days 1 and 3 following transient MCAO. a Western blot analysis of MMP-9. Treatment with MSCs downregulated the levels of MMP-9 1 and 3 days after TMCAO when compared with the PBS group. $n=4-6$ in each time point per group. $\mathbf{b}$ Zymography and quantification of active MMP-9 levels in mice brain from four groups at 1 and 3 days after tMCAO. Data are mean \pm SD. ${ }^{*} p<0.05,{ }^{* *} p<0.01,{ }^{* *} p<0.001$

selective AMPK inhibitor, compound $\mathrm{C}$ to break the AMPK phosphorylation. We found that compound $\mathrm{C}$ reduced MSC-induced AMPK phosphorylation $(p<0.05)$. The results suggested that MSC-induced change of ICAM-1 might be AMPK dependent. Thus, we conclude that MSCs diminished the expression of ICAM-1 and potentially via an AMPK-mediated signaling pathway.

\section{Discussion}

Our studies revealed that the beneficial effects of MSC on BBB after ischemia were due to the decrease of MMP-9 expression in neutrophil cells. These effects were mediated via an AMPK-dependent ICAM-1 downregulation potentially, thus alleviated neutrophil infiltration and inflammatory cytokines release in cerebral ischemic mice.

MMP-9 is confirmed to be a key protease interfering with $\mathrm{BBB}$ leakage and natural evolution of cerebral ischemia. It is produced in a prime form within cells, then activated by cleavage off the propeptide after release to extracellular space $[16,25]$. In particular, MMPs are tightly regulated at both transcriptional and post-transcriptional level by transcription factors and tissue inhibitors of MMPs (TIMPs) [26]. Moreover, it has been proposed that detrimental effects of tPA beyond the $3 \mathrm{~h}$ of stroke onset are derived from tPA's ability to activate MMP-9 [27]. This in turn contributes to the breakdown of BBB [28]. Certainly, MMP-9 has been the most widely studied MMP family member in BBB leakage, leukocyte infiltration, brain edema, and hemorrhage [14, 15]. Indeed, pro-MMP-9 is expressed almost exclusively in neutrophils in peripheral blood [16] and release to degrade the basal lamina of the endothelial cells and astrocytes locally at BBB [29, 30].

Several studies strongly suggest and demonstrate the implication of recruited leukocytes as a significant source of MMP-9 causing basal lamina degradation and $\mathrm{BBB}$ breakdown after transient ischemia [17, 31]. Neutrophils roll along and adhere to endothelium so that transmigrate through the endothelial cell barrier into the brain parenchyma to initiate the release/production of MMP-9 from resident brain cells (e.g., neurons, glial cells) to perpetuate the injury cascade [32, 33]. Increased $\mathrm{BBB}$ permeability induced by leukocyte-derived MMP-9 has been shown to correlate with peak neutrophil infiltration in ischemia-reperfusion injury $[16,29,34]$. In this study, MSCs affect neutrophil adhesion and transmigration which attenuates the upward trend of MMP-9 from filtrating neutrophils and resident cells. Modulating MMP-9 or neutrophil infiltration processes could be a target for future investigations to improve the present thrombolytic therapy for ischemic stroke. 

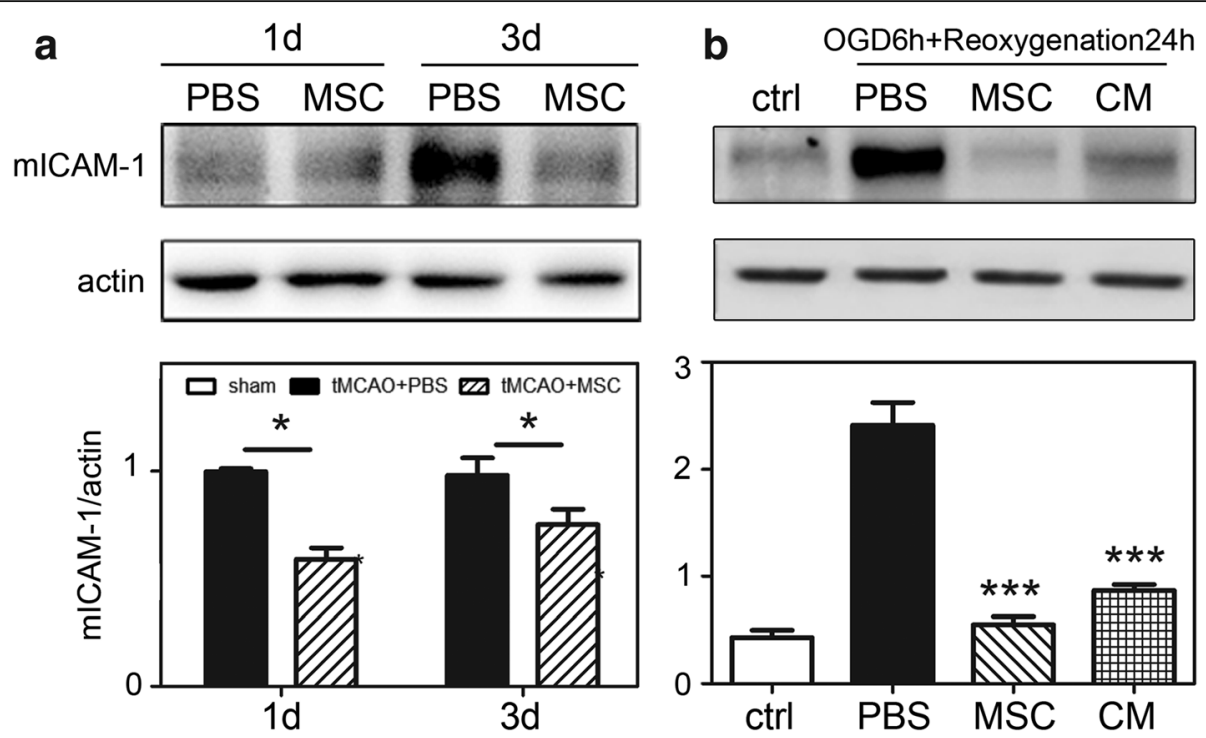

Fig. 6 MSCs might inhibit matrix metallo-proteinase-9 upregulation via reducing the expression of endogenous ICAM-1. a Western blot analysis showed the expression of ICAM-1 between PBS- and MSC-treated group at days 1 and 3 following IMCAO. $\mathbf{b}$ Western blot analysis showed the expression of mICAM-1 between (1) bEND.3 cells without OGD, (2) bEND.3 treated with PBS, (3) bEND.3 cells co-cultured with MSCs after OGD, and (4) bEND.3 cells cultured with the condition medium of MSCs after OGD in vitro. ${ }^{*} p<0.05,{ }^{* *} p<0.01,{ }^{* * *} p<0.001$

Furthermore, the recruitment of leukocytes by the vascular endothelium plays a deleterious role during multistep event in the evolution of inflammatory lesions; endothelial cell adhesion molecules serve not only as docking structures but also initiate signal cascades. In addition to facilitating leukocyte adhesion, cell adhesion molecules might also contribute to the overall proinflammatory activation of the endothelial cells [35]. In our study, endothelial cell adhesion molecules ICAM-1 is directly involved in sustaining leukocyte adhesion. These observations support the hypothesis that attenuation of observed immunomodulatory effects of MSC may contribute to the adhesion molecule expression. ICAM-1 is required for lymphocyte adhesion and, thus, plays an important role in MSC-mediated immunosuppression [36].

AMP-activated kinase (AMPK) is a highly conserved heterotrimeric kinase that functions as a metabolic switch, thereby coordinating the cellular enzymes involved in carbohydrate and fat metabolism to enable ATP conservation and synthesis [37]. AMPK is activated by conditions that increase the AMP:ATP ratio, such as exercise and metabolic stress. When the AMP:ATP ratio increases, AMPK is activated by AMPK kinase, and a conformational change is induced by combining with AMP, thereby decreasing the AMP:ATP ratio by switching off ATP-consuming pathways and switching on ATPgenerating pathways [37]. In this study, phosphorylation of AMPK in bEND.3 cells was upregulated in hypoxia but downregulated after co-culture with MSCs. We also found that inhibiting AMPK activation by compound $\mathrm{C}$ could reverse MSC-induced AMPK phosphorylation in vitro. It is generally accepted that ICAM- 1 is a NF-kB-mediated gene [38]. AMPK activation may be responsible for the inhibition of NF- $\mathrm{kB}$ activation, so we suppose that MSC inhibits the cytokine-induced expression of pro-inflammatory and adhesion molecule genes by suppressing NF- $\mathrm{kB}$ activity via AMPK activation, which needs to be further studied.

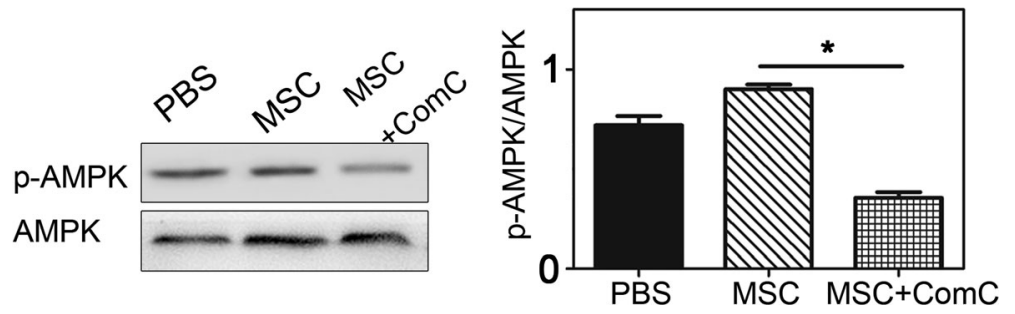

Fig. 7 MSC transplantation promoted phosphorylation of AMPK which might reduce ICAM-1 expression in vitro. Western blot showed p-AMPK and AMPK expression and quantification in PBS- and MSC-treated and MSCs plus compound C groups at $24 \mathrm{~h}$ after re-oxygenation in OGD model. Bar graph showed the quantification of $p$-AMPK/AMPK ratio. ${ }^{*} p<0.05,{ }^{* *} p<0.01,{ }^{* * *} p<0.001$ 
MSCs have been shown to have therapeutic potential in multiple disease states characterized by vascular instability. Much of the demonstrated potential of MSCs in human disease models has been shown to be due to the production of soluble factors. Some scholars have studied in traumatic brain injury (TBI) or intracerebral hemorrhage (ICH), TIMP-1, or TIMP-3 released by MSCs could stabilize BBB integrity [39, 40]. The protective effect of MSCs on BBB was possibly invoked by increased expression of tumor necrosis factor alphastimulated gene-6 (TSG-6), which may suppress the activation of the NF- $\kappa B$ signaling pathway [41]. We had considered the effects of TIMPs secreted by MSCs could reduce MMP-9 activity in ischemic BBB initially, but our in vivo results showed that, in addition to the changes in MMP-9 vitality shown by zymography, protein levels were obviously changed. Thus, there should be other secreted substances of MSCs, which severely affected the production of MMP-9 rather TIMP. Our in vitro data showed that MSC condition medium was capable of inducing ICAM-1 overexpression in bEND. 3 cells indicating that MSCs produced soluble factors to regulate the function of endothelial cells. But what MSCs secreted to decrease ICAM-1 expression in ECs was unclear at this time. In light of these findings, we suggested that secretion of certain substances MSCs attenuated the cytokine-induced expression of pro-inflammatory and adhesion molecule genes via AMPK activation. Identifying these factors is considered as the possibility of a cellfree therapeutic instead of cells. We demonstrated that ICAM-1 participation in stem cell-mediated immunesuppression would be helpful in guiding the application of clinical anti-adhesion therapies.

\section{Conclusions}

MSCs exerted a potent regulating function on endothelial ICAM-1 expression, which consequently attenuated neutrophil infiltration, MMP-9 function, and ischemia-induced BBB disruption. We highlighted the potential relationship between immunomodulatory effect and MMP-9. Our investigation revealed that MMP-9 might associate with the infiltration of neutrophils in MSC therapy after ischemia for the first time. These strategies might provide a new insight for future therapies that aim to prevent breakdown of the BBB and eventually offer therapeutic options for stroke.

\section{Abbreviations \\ BBB: Blood-brain barrier; ICAM-1: Intercellular adhesion molecule-1; MCAO: Middle carotid artery occlusion; MMP: Matrixmetallo-proteinase-9; MSCs: Mesenchymal stem cells; OGD: Oxygen-glucose deprivation; rtPA: Recombinant tissue plasminogen activator}

\section{Acknowledgements}

We thank the members of the Neuroscience and Neuroengineering Research Center for their collective support.

\section{Funding}

This study was supported by grants from the National Key Research and Development Program of China (2016YFC1300600), the National Natural Science Foundation of China (81471178, GYY; 81771251, GYY; 81771244, ZJZ), K. C. Wong Education Foundation (GYY), and the Science and Technology Commission of Shanghai Municipality (17ZR1413600, ZJZ).

Availability of data and materials

All data generated or analyzed during this study are included in this published article.

\section{Authors' contributions}

ZC was involved in the research design, experimental performances, data analysis, and drafting of the manuscript. LW and MQ participated in the cell culture and animal behavioral tests. HL helped establish the ischemic models. WL and YL participated in the cell transplantation. LW discussed the results and edited the manuscript. LD edited the manuscript. ZZ and GY took care of all aspects including the research design, data analysis, and manuscript preparation. All authors read and approve the final manuscript.

\section{Ethics approval}

Animal protocol was approved by the Institutional Animal Care and Use Committee of Shanghai Jiao Tong University, Shanghai, China. All animal procedures were performed to minimize pain or discomfort in accordance with current protocols.

\section{Competing interests}

The authors declare that they have no competing interests.

\section{Publisher's Note}

Springer Nature remains neutral with regard to jurisdictional claims in published maps and institutional affiliations.

Received: 16 January 2018 Accepted: 9 April 2018

Published online: 03 May 2018

References

1. Mathers CD, Boerma T, Ma FD. Global and regional causes of death. Br Med Bull. 2009;92:7

2. Chen $Z$, Jiang B, Ru X, Sun H, Sun D, Liu X, Li Y, Li D, Guo X, Wang W. Mortality of stroke and its subtypes in China: results from a nationwide population-based survey. Neuroepidemiology. 2017;48:95-102.

3. Pe VDS, Dubois B, Nelissen I, Rudd PM, Dwek RA, Opdenakker G. Biochemistry and molecular biology of gelatinase $B$ or matrix metalloproteinase-9 (MMP-9). Crit. Rev. Biochem. Mol. Biol. 2002;37:375-536.

4. Yang GY, Betz AL, Chenevert TL, Brunberg JA, Hoff JT. Experimental intracerebral hemorrhage: relationship between brain edema, blood flow, and blood-brain barrier permeability in rats. J Neurosurg. 1994;81:93.

5. Gasche Y, Copin JC, Sugawara T, Fujimura M, Chan PH. Matrix metalloproteinase inhibition prevents oxidative stress-associated bloodbrain barrier disruption after transient focal cerebral ischemia. J Cereb Blood Flow Metab. 2001;21:1393-400

6. Clark AW, Krekoski CA, Bou SS, Chapman KR, Edwards DR. Increased gelatinase A (MMP-2) and gelatinase B (MMP-9) activities in human brain after focal ischemia. Neurosci Lett. 1997;238:53-6.

7. Rosell A, Ortegaaznar A, Alvarezsabín J, Fernándezcadenas I, Ribó M, Molina CA, Lo EH, Montaner J. Increased brain expression of matrix metalloproteinase-9 after ischemic and hemorrhagic human stroke. Stroke. 2006;37:1399-406.

8. Ji HH, Lucero J, Abumiya T, Koziol JA, Copeland BR, Zoppo GJD. Matrix metalloproteinases increase very early during experimental focal cerebral ischemia. J Cereb Blood Flow Metab. 1999;19:624-33.

9. Anthony DC, Ferguson B, Matyzak MK, Miller KM, Esiri MM, Perry VH. Differential matrix metalloproteinase expression in cases of multiple sclerosis and stroke. Neuropathol Appl Neurobiol. 1997;23:406.

10. Suzuki Y, Nagai N, Umemura K, Collen D, Lijnen HR. Stromelysin-1 (MMP-3) is critical for intracranial bleeding after t-PA treatment of stroke in mice. J Thromb Haemost. 2007;5:1732.

11. Yang Y, Estrada EY, Thompson JF, Liu W, Rosenberg GA. Matrix metalloproteinase-mediated disruption of tight junction proteins in cerebral 
vessels is reversed by synthetic matrix metalloproteinase inhibitor in focal ischemia in rat. J Cereb Blood Flow Metab. 2007;27:697-709.

12. Svedin P, Hagberg H, Sã Vman K, Zhu C, Mallard C. Matrix metalloproteinase-9 gene knock-out protects the immature brain after cerebral hypoxia-ischemia. J Neurosci Official J Soc Neurosci. 2007;27:1511-8.

13. Dejonckheere $E$, Vandenbroucke RE, Libert C. Matrix metalloproteinases as drug targets in ischemia/reperfusion injury. Drug Discov Today. 2011;16:762.

14. Adibhatla RM, Hatcher JF. Tissue Plasminogen Activator (tPA) and Matrix Metalloproteinases in the Pathogenesis of Stroke: Therapeutic Strategies. CNS \& neurological disorders drug targets. 2008;7(3):243-53.

15. Cunningham LA, Wetzel M, Rosenberg GA. Multiple roles for MMPs and TIMPs in cerebral ischemia. Glia. 2005;50:329-39.

16. Tang Y, Xu H, Du XL, Lit L, Walker W, Lu A, Ran R, Gregg JP, Reilly M, Pancioli A. Gene expression in blood changes rapidly in neutrophils and monocytes after ischemic stroke in humans: a microarray study. J Cereb Blood Flow Metab. 2006;26:1089.

17. Turner RJ, Sharp FR. Implications of MMP9 for blood brain barrier disruption and hemorrhagic transformation following ischemic stroke. Front Cell Neurosci. 2016;10:56.

18. Dominici ML, Le Blanc K, Mueller I, Slaper-Cortenbach I, Marini FC, Krause DS, Deans RJ, Keating A, Prockop DJ, Horwitz EM. Minimal criteria for defining multipotent mesenchymal stromal cells. The International Society for Cellular Therapy position statement. Cytotherapy. 2009:8:315.

19. Rowart P, Erpicum P, Detry O, Weekers L, Gregoire C, Lechanteur C, Briquet $A$, Beguin $Y$, Krzesinski JM, Jouret F. Mesenchymal stromal cell therapy in ischemia/reperfusion injury. J Immunol Res. 2015;2015:602597.

20. Zhang H, Sun F, Wang J, Xie L, Yang C, Pan M, Shao B, Yang GY, Yang SH, Zhuge Q. Combining injectable plasma scaffold with mesenchymal stem/ stromal cells for repairing infarct cavity after ischemic stroke. Aging Dis. 2017;8:203-14

21. Rowart P, Erpicum P, Detry O, Weekers L, Grégoire C, Lechanteur C, Briquet A, Beguin Y, Krzesinski JM, Jouret F. Mesenchymal stromal cell therapy in ischemia/reperfusion injury. J Immunol Res. 2015;2015:602597.

22. Tang G, Liu Y, Zhang Z, Lu Y, Wang Y, Huang J, Li Y, Chen X, Gu X, Wang Y. Mesenchymal stem cells maintain blood-brain barrier integrity by inhibiting aquaporin-4 upregulation after cerebral ischemia. Stem Cells. 2014;32:3150-62.

23. Horwitz EM, Gordon PL, Koo WKK, Marx JC, Neel MD, Mcnall RY, Muul L, Hofmann T. Isolated allogeneic bone marrow-derived mesenchymal cells engraft and stimulate growth in children with osteogenesis imperfecta: implications for cell therapy of bone. Proc Natl Acad Sci U S A. 2002;99:8932.

24. Liu Y, Tang G, Li Y, Wang Y, Chen X, Gu X, Zhang Z, Wang Y, Yang GY. Metformin attenuates blood-brain barrier disruption in mice following middle cerebral artery occlusion. J Neuroinflammation. 2014;11:177.

25. Vandooren J, Van DJ, Opdenakker G. On the structure and functions of gelatinase B/matrix metalloproteinase-9 in neuroinflammation. Prog Brain Res. 2014;214:193.

26. Clark IM, Swingler TE, Sampieri CL, Edwards DR. The regulation of matrix metalloproteinases and their inhibitors. Int J Biochem Cell Biol. 2008;40:1362-78.

27. Wang X, Lee SR, Arai K, Lee SR, Tsuji K, Rebeck GW, Lo EH. Lipoprotein receptor-mediated induction of matrix metalloproteinase by tissue plasminogen activator. Nat Med. 2003;9:1313.

28. Chaturvedi M, Kaczmarek L. MMP-9 inhibition: a therapeutic strategy in ischemic stroke. Mol Neurobiol. 2014;49:563-73.

29. Justicia C, Panés J, Solé S, Cervera A, Deulofeu R, Chamorro A, Planas AM. Neutrophil infiltration increases matrix metalloproteinase-9 in the ischemic brain after occlusion/reperfusion of the middle cerebral artery in rats. J Cereb Blood Flow Metab. 2003:23:1430-40.

30. Gidday JM, Gasche YG, Copin JC, Shah AR, Perez RS, Shapiro SD, Chan PH, Park TS. Leukocyte-derived matrix metalloproteinase-9 mediates blood-brain barrier breakdown and is proinflammatory after transient focal cerebral ischemia. Am J Physiol Heart Circ Physiol. 2005;289:H558.

31. Rosell A, Cuadrado E, Ortegaaznar A, Hernándezguillamon M, Lo EH, Montaner J. MMP-9-positive neutrophil infiltration is associated to bloodbrain barrier breakdown and basal Lamina type IV collagen degradation during hemorrhagic transformation after human ischemic stroke. Stroke. 2008:39:1121-6.

32. Wang $\mathrm{Q}$, Doerschuk $\mathrm{CM}$. The signaling pathways induced by neutrophilendothelial cell adhesion. Antioxid Redox Signal. 2002:4:39.

33. Schnoor M, Parkos CA. Disassembly of endothelial and epithelial junctions during leukocyte transmigration. Frontiers Biosci J Virtual Library. 2008;13:6638.
34. Sandoval KE, Witt KA. Blood-brain barrier tight junction permeability and ischemic stroke. Neurobiol Dis. 2008:32:200

35. Greenwood J, Etienne-Manneville S, Adamson P, Couraud PO. Lymphocyte migration into the central nervous system: implication of ICAM-1 signalling at the blood-brain barrier. Vasc Pharmacol. 2002;38:315-22.

36. Ren G, Zhao X, Zhang L, Zhang J, L'Huillier A, Ling W, Roberts Al, Le AD, Shi S, Shao C, Shi Y. Inflammatory cytokine-induced intercellular adhesion molecule-1 and vascular cell adhesion molecule-1 in mesenchymal stem cells are critical for immunosuppression. J Immunol. 2010;184:2321-8.

37. Hardie DG, Carling D, Carlson M. The AMP-activated/SNF1 protein kinase subfamily: metabolic sensors of the eukaryotic cell? Annu Rev Biochem. 1998;67:821

38. Tak PP, Firestein GS. NF-kappaB: a key role in inflammatory diseases. J Clin Investig. 2001;107:7.

39. Menge T, Zhao Y, Jing Z, Wataha K, Geber M, Zhang J, Letourneau P, Redell J, Li S, Jing W. Mesenchymal stem cells regulate blood brain barrier integrity in traumatic brain injury through production of the soluble factor TIMP3. Sci Transl Med. 2012;4:161ra150

40. Miao M, Zhao JX, Fang SS. The expression of TIMP-1 in perihematoma and relationship of TIMP-1 to brain edema formation after intracerebral hemorrhage in rats. Pract J Med Pharm. 2011;28(3):259-61.

41. Min C, Li X, Xin Z, He X, Lai L, Liu Y, Zhu G, Wei L, Hui L, Fang Q. The inhibitory effect of mesenchymal stem cell on blood-brain barrier disruption following intracerebral hemorrhage in rats: contribution of TSG-6. J Neuroinflammation. 2015;12:1-14.

\section{Ready to submit your research? Choose BMC and benefit from:}

- fast, convenient online submission

- thorough peer review by experienced researchers in your field

- rapid publication on acceptance

- support for research data, including large and complex data types

- gold Open Access which fosters wider collaboration and increased citations

- maximum visibility for your research: over $100 \mathrm{M}$ website views per year

At BMC, research is always in progress.

Learn more biomedcentral.com/submissions 